\title{
What and How Do Cancer Systems Biologists Explain?
}

\author{
Anya Plutynski and Marta Bertolaso*†
}

In this article, we argue, first, that there are very different research projects that fall under the heading of "systems biology of cancer." While they share some general features, they differ in their aims and theoretical commitments. Second, we argue that some explanations in systems biology of cancer are concerned with properties of signaling networks (such as robustness or fragility) and how they may play an important causal role in patterns of vulnerability to cancer. Further, some systems biological explanations are compelling illustrations of how "top-down" and "bottom-up" approaches to the same phenomena may be integrated.

1. Introduction. "Systems biology" has become an extremely popular field of study - one that has attracted the attention of funding agencies and institutions around the globe. This is especially so in the context of medicine. Cancer systems biology, for example, is presented as a more "systematic" approach to such a complex disease. However, those who adopt this label are not concerned with the same family of questions and do not necessarily endorse one common set of theoretical commitments or methods.

Our project in this article is to examine what - if anything - is distinctive about explanations offered in the domain of cancer systems biology. In service of this aim, we first briefly discuss the historical origins of the field. We follow a twofold classification of systems biology approaches, drawing on O'Malley and Dupré (2005): a "pragmatic" and a "systems theoretical" approach. Pragmatic approaches are concerned largely with prediction, or what

*To contact the authors, please write to: Anya Plutynski, Department of Philosophy, 1 Brookings Hall, Washington University, St. Louis, MO 63130; e-mail: aplutyns@wustl .edu. Marta Bertolaso, Università Campus Bio-Medico di Roma; e-mail: mbertolaso @unicampus.it.

$\dagger$ The article was jointly developed by both authors and the thesis commonly shared. Anya Plutynski was mainly responsible for writing sec. 2, and Marta Bertolaso, for writing secs. 1 and 4.

Philosophy of Science, 85 (December 2018) pp. 942-954. 0031-8248/2018/8505-0017\$10.00

Copyright 2018 by the Philosophy of Science Association. All rights reserved. 
is often described as "translational" medicine: they aim at developing new prognostic and diagnostic tests and designing targeted interventions. In contrast, "systems theoretical" approaches are concerned with identifying general principles of systems, such as structural features of regulatory pathways or signaling networks that play a role in "tipping the balance" toward a cancerous phenotype. In addition, one central aim of the latter approach is to understand features of such systems that enable the evolution of resistance to treatment.

There is another way of characterizing "systems" approaches, which we also find fruitful (Bruggeman and Westerhoff 2007): a "bottom up" versus "top down" approach. Many systems biological explanations fall somewhere in the middle. That is, they involve integration of a mechanistic understanding of cell and molecular processes with "macro-" or "systems level" approaches. This distinction is helpful in that it illuminates how systems biological explorations of cancer are often multiscale, building "up" from biochemistry and cell and molecular biology and, simultaneously, moving "down" from structural or network models into the realizers of these patterns and their common features (for similar views, see also Fagan 2012; O'Malley et al. 2014; Green, Fagan, and Jaeger 2015).

2. A Brief History: Systems Biology in Cancer. Many scientists characterize their work as "cancer systems biology." Not all of them share the same theoretical commitments or aims. Indeed, given the competitive funding environment for cancer research, and the rhetorical power of talk of cancer as a "systems" disease, some measure of skepticism is in order regarding the unity of "cancer systems biology" as a field of inquiry. The label's popularity is in part a product of institutional and economic forces and in part also a product of a broader set of transitions in biological sciences, growing out of the expansion of biotechnology, the genomes projects, and the increasing emphasis on interdisciplinarity in the twenty-first century.

One indicator of how a systems approach has been institutionalized can be found in a National Research Council (2009) report. In July 2008 the National Institutes of Health (NIH), National Science Foundation, and Department of Energy asked the National Research Council's Board on Life Sciences to "examine the current state of biological research in the United States and recommend how best to capitalize on recent technological and scientific advances that have allowed biologists to integrate biological research findings, collect and interpret vastly increased amounts of data, and predict the behavior of complex biological systems" (11). The board convened a committee to take on this assignment. This resulted in the aforementioned report. The committee made a number of recommendations. First, they encouraged funding research that links engineering, mathematics, computer science, and biology. Second, they emphasized similarities across biological systems at different 
scales and encouraged identification and exploration of common properties of biological and engineered systems, such as "fragility, robustness, complexity, and organizing principles." The report led to a new funding program at the NIH in integrative or systems biology.

Since 2008, institutes for, schools of, and centers for systems biology have been created at many of the major research institutions in the United States (Stanford, Harvard, New York University, Columbia, University of Massachusetts, Washington State, etc.) and internationally (Italy, Germany, Hungary, France, Denmark, Austria, Switzerland, the United Kingdom, Russia, India, China, Pakistan, Japan, and Australia). The NIH founded an Integrative Cancer Biology Program, which now funds 12 centers for Cancer Systems Biology. The stated aims of these programs vary. What, apart from the label, ties all these programs together? The stated of aims of many of these institutions, as presented on websites and in papers, include the following common characteristics:

- All such programs emphasize interdisciplinarity. They host scholars from diverse disciplines, such as biostatistics, computational biology, engineering, computer science, genetics and genomics, and cell and molecular biology. They also emphasize interdisciplinary methods and, particularly, development and use of new technology, for example, highthroughput experimental platform technologies.

- They are data driven, often involving "big data." In particular, the rise of the cancer genomics, epigenomics, proteomics, and transcriptomics projects has led to a vast amount of information that needs to be curated, organized, and mined to generate network models, for instance, documenting the co-occurrence of mutations or gene products in various cancer subtypes. These are collected and collated by national and international organizations, such as the Cancer Genome Database, or the Cancer Genome Atlas.

- They are concerned with modeling: simulation models, mathematical models of various sorts, network models, or multiscale models (Deisboeck et al. 2011). Such models may be used to generate hypotheses, integrate data, or simply provide visual representations of otherwise overwhelming amounts of statistical data, such as representations of genes frequently mutated in this or that cancer type or dynamic spatial models of vast interconnected cell signaling pathways associated with outcomes and processes in cancer at very different scales.

The vast majority of this research is concerned with diagnosis and treatment: developing "targeted" drugs or better classifying cancer types and subtypes so as to arrive at more precise, or "personalized," therapy. This work might be characterized as more "pragmatic" in character, and it uses 
data-driven methods that, for instance, draw on thousands of samples from tumor biopsies and sequences of these samples to generate databases of cancer biomarkers (genomic, epigenomic, transcriptomic, proteomic), in service of better predictions about the behavior of different subtypes of cancer or likely drug targets. Much of this work uses machine learning, bioinformatics, and network modeling.

In contrast, some work in cancer systems biology is less focused on the pragmatic applications of the research than on arriving at some general theoretical principles about the dynamics and topography of complex systems, taking cancer as a case in point. This distinction nicely coincides with the more general distinction made by O'Malley and Dupré: "Systems biology is best understood as consisting of two streams. One, which we shall call 'pragmatic systems biology', emphasizes large-scale molecular interactions; the other, which we shall refer to as 'systems-theoretic biology', emphasizes system principles" $(2005,1270)$. Some systems theoretical biologists argue that there are common structural features of networks that both explain and predict cancers' behaviors. Such structural features are sometimes called "motifs." In general, systems theoretical biologists are concerned with how general features of networks explain or predict the behavior of complex biological systems. More precisely, in this field any network property (e.g., network robustness or degree distribution) is both an explanandum and explanans (cf. Huneman 2017).

For example, some systems biologists have argued that there may be distinctive structural features of networks of signaling molecules controlling cell birth and death that make us far less vulnerable to cancer than we might otherwise be. Scale-free networks are networks that have a degree distribution that follows a power law, where a network's degree distribution is the network-wide proportion of edges connected to $k$ other nodes. So, scale-free networks have many nodes connected to only one or a few other nodes and only a few nodes connected to many other nodes (hubs). Scale-free networks have a number of core properties, what systems biologists call "distributed robustness": "many parts of a system contribute to system function, but all of these parts have different roles. When one part fails or is changed through mutations, the system can compensate for this failure, but not because a 'back-up' redundant part takes over the failed part's role" (Wagner 2005, 177). Robust systems maintain function despite perturbation, where perturbation might mean elimination or disruption of the activity of nodes.

In scale-free networks, one can eliminate many such nodes without disrupting function. However, if one disrupts several hubs, then function is destabilized. Thus, scale-free networks are "robust" generally but are vulnerable to disruption of highly connected nodes. All things considered, then, scale-free networks may be considered to be relatively robust to insult, in comparison to randomly distributed networks. Once several hubs are af- 
fected, they can quickly collapse. Indeed, this may be what we see in cancer. In the case of cancer, there are core "drivers," or hubs, that affect many downstream regulatory functions in the cell, including but not limited to advancing the cell cycle (and thus initiating or halting cell division), initiating cell death, and transferring such signals from outside to inside the cell.

Some of the genes most commonly affected in cancer are hubs in networks of signaling cascades that affect cell birth and death. The scale-free network structure of these signaling pathways may explain (in part) how we are vulnerable to cancer, as well as why we do not get cancer more often. When many hubs are disrupted, there is destabilization of function; but there is also robustness to insult, insofar as scale-free structures prevent disruption of cell functions (Alon 2007; Barillot et al. 2012).

To be sure, much of this discussion is very theoretical and difficult to establish empirically. In part for these reasons, not everyone is optimistic about this general project and method. Robert Weinberg, one of the most accomplished contributors to cancer research, has expressed skepticism about the ambitions of systems biology: "The currently embraced notion is that a complex system can only be understood if all of its moving parts are analyzed in one sweeping overview.... The data that we now generate overwhelm our abilities of interpretation, and the attempts of the new discipline of 'systems biology' to address this shortfall have to date produced few insights into cancer biology beyond those revealed by simple, homegrown intuition. The coupling between observational data and biological insight is frayed if not broken" (Weinberg 2014, 270). Is there good reason for Weinberg's skepticism of systems biology? Below we consider some examples of systems biological approaches to explaining various features of cancer. Our goal is to say what is distinctive, if anything, about such approaches, as well as to speak to whether Weinberg's skepticism about the project is warranted.

3. Examples: Network Robustness and Network Topology. Let us turn to some successful and representative examples of systems biology of cancer. One project central to cancer systems biology is mapping out networks of biochemical pathways that play important roles in behaviors typical of cancer cells. For instance, one way in which cancer cells develop the ability to grow without limit is via autocrine signaling (self-signaling loops), where mitogens (molecules that activate growth) are produced excessively, or bound excessively. The products of many so-called oncogenes, such as $s r c$, act as a mitogen. The ERBB (also known as HER) family or epidermal growth factor receptor (EGFR) family consists of four structurally related receptor tyrosine kinases - receptors for signaling molecules that play a role in cell division. They are part of a complex signaling network that is essential in growth and development. They also play a role in cancer. 
Excessive EGFR/ERBB signaling is common in a wide variety of solid tumors. Amplification of EGFR expression can be found in $20 \%-30 \%$ of metastatic breast lesions (Slamon et al. 1987) and in small fractions of several types of carcinoma (e.g., head and neck cancer and brain tumors; Ekstrand et al. 1991). The ERBB family is a classic case of pathway degeneration, which occurs when genes are duplicated in evolution, and these duplicated genes acquire some new functional specializations but conserve the original function in part. So, in this way, the associated function is robust: redundancy enables the system as a whole to perform the same function. This is the case for ERBB.

The robustness of this gene family in part explains why therapies targeting EGFR are thwarted: coactivation of alternate kinases that have partial overlap with EGFR can compensate when one particular subpart is compromised. In other words, the robustness of the network in part explains both the significance of EGFR disruption in cancer and the evolution of chemotherapy resistance to drugs that target the ERBB family. The robustness of the network in the latter case constitutes the explanans, rather than the explanandum. Of course, the way in which an appeal to the robust structure of the network does explanatory work depends on how we specify the explanandum (cf. Craver 2016).

When determining what roles ERBB plays in cancer, we might ask very different questions:

- What is the function of this complex and how is its mechanistic basis realized?

- How does disruption of that function play a role in cancer progression?

- What features of this signaling complex (and its relation to other signaling systems in the cell) make its disruption particularly likely to lead to cancer? Is this part of a larger network?

- What is the spatial organization of this network, and how does this organization contribute to its dynamic patterns of expression or regulatory functions or role in dysfunctional outcomes?

- How do drugs that target the ERBB family fail?

- Are drugs that target one or more members of this family likely to fail, and why?

- Are there specific structural features of the network that make drugs likely to fail?

In elaborating answers to the first two of these questions, we might appeal to mechanistic information. The last four questions, however, require appeal to more general systems theory. Answering all such questions will require integration of information from cell and molecular biology, systems theory, gene regulatory network modeling, computational biology, and evolution- 
ary and developmental biology. Cell and molecular biology can inform us about ERBB functions and their mechanistic realization. Such explanations are causal and functional explanations. In contrast, systems theoretical approaches can help us illuminate relevant features of ERBB network structure, which may contribute to its robustness, its role in our vulnerability to cancer, and its role in the evolution of chemotherapy resistance. In this way, systems approaches bridge the gap between more reductive, mechanistic approaches to cancer and evolutionary developmental biology. The latter approaches help explain why certain kinds of circuitry and network structures are so common, across a variety of species (see, e.g., Levine and Davidson 2005) and how they come to play a role in cancer.

Cell division in healthy tissue is usually tightly regulated. This is traceable to our evolutionary history. When the first multicellular organisms evolved, cells needed to acquire the capacity to cooperate - that is, limit growth, share resources, and be responsive to intercellular signals. As Amit, Wides, and Yarden $(2007,1)$ put it, "To precisely coordinate and integrate cellular decisions such as proliferation, differentiation and apoptosis, metazoans developed a set of information relay systems." Disruption of such cell signaling pathways, particularly associated with growth, is one common feature, or hallmark, of cancer cells. Many of the same hub genes are associated with several of these pathways - they are pleiotropic in their effects (see, e.g., Weinberg 2013, chap. 6, for discussion). Gene regulatory network modelers call genes and associated subcircuits that play important roles in locking down key functions in the cell "kernels" (Levine and Davidson 2005).

Such hubs play an important functional and structural role in cancer progression. The structure of such networks helps explain consistent patterns in the dynamics of cancer progression, as well as how and why some cancers are either more or less resilient to insult (i.e., chemotherapy). Taking a "network perspective" on ERBB helps characterize this "Achilles heel" in cancer; that is, it helps explain how and why ERBB is so frequently disrupted and so difficult to target:

The ERBB family actually represents a bona fide biological network that obeys the principles of network theories. Interestingly, biological networks tend to adopt a scale-free architecture. ... The increased stability of such networks comes with a cost that translates into fragility: essential hubs might be 'Achilles heels'... . The ERBB system incorporates many network features, such as modularity and multiple control loops. As for other networks, the ERBB network displays two steady states (bistability), and ligands transiently switch it from OFF to ON. Several oncogenic mutations bias a prolonged ON state. For example, by favoring ERBB2-containing heterodimers and escaping negative feedback regulation, pathological $E R B B 2$ amplification creates a hub that enhances signaling while exposing 
pharmacological susceptibilities. ... Network plasticity and robustness are manifested repeatedly following drug intervention, as reflected by the emergence of drug resistance. . . Biological networks show fragility in the face of simultaneous perturbations (for example, combination therapy). Another uncommon perturbation is exemplified by monoclonal-antibody-mediated recruitment of the immune system. Careful analyses of the network may reveal other tumor-specific vulnerabilities and addictions that are amenable for pharmacological interventions. (Yarden and Pines 2012, 560).

In sum, what explains the ERBB complex's unique role in cancer, and robustness to single agent chemotherapy, is the structure of the network of which it is a part.

The ERBB complex thus provides an illustration of several general principles in systems theory. Namely, the very properties of cell signaling pathways that enable robustness and evolvability (modularity, scale-free structure) can become a site of vulnerability, and redundancy can both be an advantage and pose a challenge in treatment contexts. Systems biologists identify common elements of such networks and the roles they play in various stages of progression in cancer. They first identify associations between particular genes mutated in cancer and then build a network model, a representation of both the organizational structure and dynamics of gene expression over time, as a tumor progresses. For instance, Sadeghi et al. (2016) distinguished the most important transcription factors affected in primary prostate tumor samples (HOXD10, BCL2, and PGR) from those playing a central role in the metastatic state (STAT3, JUN, and JUNB). Before their analysis, a number of genes had been reported as involved in prostate cancer progression, being down regulated in some studies and overexpressed in other studies (see also Bo and Tucker 2015; Nazarieh et al. 2016). What the network model achieved was identification of central hubs and likewise targets for intervention at different stages in progression.

More generally, molecular systemic models of the cell connect molecular agents, growth factors, and cytokines into an endogenous molecular-cellular network characterized by these distinctive interactions and associated vulnerabilities. The topological features of these complex networks can be used to model the dynamics of progression typical in cancer. A nonlinear system is a system in which the change of the output is not proportional to the change of the input and may appear chaotic. Complex networks have multiple stable states (where a state is a pattern of activation of elements and variables). These stable states are called "attractors," which reside within a "functional landscape" of the network. The state space is the total set of possible network states, plus the transitions and trajectories between them. The particular architecture of the network determines how movement through 
the state space is constrained. In other words, the network structure of such systems helps us identify how and when mutations to sets of genes, such as the ERBB family, are likely to lead to cancer.

According to some proponents of systems theory, changes in the cellular phenotype underlying progression to metastasis may result from exactly these transitions within entire genome-wide gene regulatory networks. Mutations can lead to molecular signals that contribute to the shift of the system toward an attractor (eventuating in a tumor; Huang and Ingber 2007; Huang, Ernberg, and Kauffman 2009). Cancer, from this perspective, is seen as a robust state of the endogenous cellular network (Ao et al. 2008). That is, our vulnerability to cancer is an intrinsic property of the cells - one inherited from the developmental and evolutionary history of the organism. For example, the transition from mesenchyme to epithelial cells is one that all epithelial cells undergo during development; cancer shows that the capacity to "return" to this phenotype is retained. An epithelial-mesenchymal transition encompasses, in a broad sense, all changes in cell morphology from epithelioid to mesenchymal correlated to the increased motility of cancer cell lines. In the attractor landscape perspective, the mesenchyme phenotype is considered a distinct cellular program, a "stable state," to which cancer cells return by reacquiring those same gene expression profiles typical of earlier developmental stages. To the extent that cells have this potential, these "embryonic" attractors remain present in adulthood (Laforge et al. 2005) and effectively function as "tumor attractors" (Fogarty, Kessler, and WechslerReya 2005). In this way, ontogeny provides oncogenesis with a starting point (Huang and Ingber 2007; Ingber 2008). In other words, from a systems perspective, we may view the malignant phenotype as one of the states potentially existing in the cell. Preexisting attractors explain the ease with which random mutations can quickly produce features typical of earlier developmental stages.

There are several such "endogenous" networks - networks that are typical or normally activated in healthy or developing tissue - that may be activated in cancer. For instance, tumors co-opt the wound-healing response in order to induce the stroma they require for maintenance and growth. As Dvorak originally suggested (1986), cancers are "wounds that do not heal." Tumors share properties with healing wounds. Vascular permeability factor (VPF, subsequently renamed vascular endothelial growth factor [VEGF]) is a tumor product. The sequence of events associated with cancer progression (increased vascular permeability; extravasation of plasma, fibrinogen, and other plasma proteins; activation of the clotting system outside the vascular system; etc.) also takes place in a variety of important inflammatory diseases that involve cellular immunity (Dvorak 2015). One can model such cases as an example of a network of inter- and intracellular signaling, which achieves a stable state, via production of gradients of growth factors, modulation of 
the permeability of membranes, and generation of new structures (in this case, angiogenesis).

Networks such as this are sensitive to critical thresholds. According to the Autostabilization-Selection Model (Laforge et al. 2005), a gradual accumulation of many small alterations in signaling molecules within and between cells can lead to a shift in an ordinarily stable state. Laforge's model incorporates experimental data from gene expression studies and traces the shift in stability to the modulation of the concentration of transcriptional regulators in the cell in relation to differentiation. It also takes into account similarities with models pertaining to the theories of morphogenesis. On this model, the process of cancer progression enacts, in several respects, a reversal of morphological development. Tissue formation is not the result of a single type of cellular change or specific mechanistic interaction but involves multiple complementary molecules that exert various effects on tissue organization. An imbalance in these factors can lead to tissue disorganization and invasive growth.

Several systems biological explanations are bottom-up as much as they are top-down. These two consolidated, conceptually different approaches (Van Laere, Dirix, and Vermeulen 2016) thus find integration in cancer systems biology. "Bottom-up" approaches reveal upstream signaling pathways based on gene expression profile changes. "Top-down" approaches translate gene expression changes, or any other molecular profile, into downstream biological responses. Top-down gene clustering can assist in finding sets of coregulated genes. "Network enrichment" is a technique that establishes which pathways and processes are relevant to a given outcome and how they are associated with each other in the network. Networks may also be built from molecular data (this is "network inference"; Bansal et al. 2007). The bottom-up approach is better suited for identifying pathways, whereas the top-down approach is more appropriate to evaluate biological processes, functional and dysfunctional (Van Laere et al. 2016).

For example, a recent study inferred a set of 33 transcription factors associated with gene signatures of several known phenotypes involved in small cell lung cancer (SCLC; Udyavar et al. 2017). Udyavar et al. built a network simulation, incorporating all known involved pathways. Simulations predicted that the network will settle into attractors, or transcription factors expression patterns, that correlate with phenotypes. The strong correlation with observed outcomes suggested that transcription factors network dynamics underlie the emergence of heterogeneous SCLC phenotypes. The model also predicted a hybrid phenotype that was confirmed by experimental activity (with the flow cytometry technique). This explained several clinical observations, especially treatment failures, convergence under treatment of different tumor cells on a single phenotype, and wild phenotypic heterogeneity. 
More researchers are starting to combine top-down and bottom-up approaches to build systems biological explanations of cancer phenomena. According to Udyavar et al., these approaches "should be generally useful in uncovering underlying mechanisms" $(2017,1072)$. Thus, we can see how Weinberg's skepticism might be addressed: the kinds of questions systems biologists ask, and the strategies by which they answer such questions, are different from those typical in the cell and molecular biology of cancer but complementary to them. Such approaches involve drawing on vast amounts of data, building network models, and generating hypotheses about mechanisms. So, this different approach asks (and answers) a different array of questions about cancer, focusing less on uncovering specific mechanisms than on dynamic structural relationships of networks of signaling molecules and genes associated with cancer progression.

4. The Upshot. What then is distinctive about these explanatory projects? There is nothing here that contradicts the idea that many biological explanations concern the identification and decomposition of mechanisms into their parts and processes (see, e.g., Brigandt, Green, and O'Malley 2017). However, systems biological approaches identify different target explananda than is typical of mechanistic explanations and draw on different theoretical frameworks and methods. They are concerned with general patterns of stability and instability in the dynamics of cancer progression. This requires placing cell intrinsic mechanisms associated with cancer initiation and progression in a larger context, at a variety of temporal and spatial scales, from the cell-signaling networks active in wound healing to evolutionary and developmental history. In this way, they integrate top-down and bottom-up perspectives on the same phenomena.

For instance, we argued that the structural features of certain networks explain both their resistance to and subjection to insult. This is not at odds with mechanistic description but both supplementary to and more comprehensive than mechanistic explanations, in that such accounts contextualize the role(s) of mechanisms in a larger system and sometimes show them to be instances of more general patterns. As Brigandt et al. argue, "what makes research ... distinctively systems-biological stems from the fact that large networks are screened to . . . reveal both common and uncommon elements of biological design. . . . Abstract organizational schemes . . . and design principles, transcend the organization of a single mechanism, and even a single species" $(2017,365)$.

We have extended this analysis into the realm of cancer research. We have argued first that cancer systems biology is a kludge; there are many different research projects that fall under this heading. While they share many features, they differ in their central aims and theoretical commitments. We argued that what is distinctive about explanations in cancer systems biology is 
that at least some aspire to offer top-down, structural explanations that appeal to the robust features of networks. In contrast, others appear to integrate top-down and bottom-up approaches.

\section{REFERENCES}

Alon, Uri. 2007. "Network Motifs: Theory and Experimental Approaches." Nature Reviews Genetics 8 (6): 450-61.

Amit, Ido, Ron Wides, and Yosef Yarden. 2007. "Evolvable Signaling Networks of Receptor Tyrosine Kinases: Relevance of Robustness to Malignancy and to Cancer Therapy." Molecular Systems Biology 3 (1): 1-14.

Ao, Ping, David Galas, Leroy Hood, and Xiaomei Zhu. 2008. "Cancer as Robust Intrinsic State of Endogenous Molecular-Cellular Network Shaped by Evolution.” Medical Hypotheses 70 (3): 678-84.

Bansal, Mukesh, Vincenzo Belcastro, Alberto Ambesi-Impiombato, and Diego Di Bernardo. 2007. "How to Infer Gene Networks from Expression Profiles."Molecular Systems Biology 3 (1): 78.

Barillot, Emmanuel, Laurence Calzone, Philippe Hupe, Jean-Philippe Vert, and Andrei Zinovyev. 2012. Computational Systems Biology of Cancer. Boca Raton, FL: CRC.

Bo, Valeria, and Allan Tucker. 2015. "Integrating Gene Regulatory Networks to Identify CancerSpecific Genes." In Proceedings of the American Medical Informatics Association Joint Summits on Translational Science Proceedings, 21-25. Bethesda, MD: AMIA.

Brigandt, Ingo, Sara Green, and Maureen A. O'Malley. 2017. "Systems Biology and Mechanistic Explanation." In The Routledge Handbook of Mechanisms and Mechanical Philosophy, ed. Stuart Glennan and Phyllis Illari. London: Routledge.

Bruggeman, Frank J., and Hans V. Westerhoff. 2007. "The Nature of Systems Biology.” Trends in Microbiology 15 (1): 45-50.

Craver, Carl F. 2016. "The Explanatory Power of Network Models.” Philosophy of Science 83 (5): 698-709.

Deisboeck, Thomas S., Zhihui Wang, Paul Macklin, and Vittorio Cristini. 2011. "Multiscale Cancer Modeling." Annual Review of Biomedical Engineering 13:127-55.

Dvorak, Harold F. 1986. "Tumors: Wounds That Do Not Heal." New England Journal of Medicine 315 (26): $1650-59$.

—. 2015. "Tumors: Wounds That Do Not Heal; Redux." Cancer Immunology Research 3 (1): $1-11$.

Ekstrand A. J., C. D. James, W. K. Cavenee, B. Seliger, R. F. Pettersson, and V. P. Collins. 1991. "Genes for Epidermal Growth Factor Receptor, Transforming Growth Factor $\alpha$, and Epidermal Growth Factor and Their Expression in Human Gliomas in Vivo." Cancer Research 51:216472 .

Fagan, Melinda Bonnie. 2012. "Waddington Redux: Models and Explanation in Stem Cell and Systems Biology." Biology and Philosophy 27 (2): 179-213.

Fogarty, M. P., J. D. Kessler, and R. J. Wechsler-Reya. 2005. "Morphing into Cancer: The Role of Developmental Signaling Pathways in Brain Tumor Formation." Journal of Neurobiology 64 (4): $458-75$.

Green, Sara, Melinda Fagan, and Johannes Jaeger. 2015. "Explanatory Integration Challenges in Evolutionary Systems Biology." Biological Theory 10 (1): 18-35.

Huang, Sui, Ingemar Ernberg, and Stuart Kauffman. 2009. "Cancer Attractors: A Systems View of Tumors from a Gene Network Dynamics and Developmental Perspective." Seminars in Cell and Developmental Biology 20 (7): 869-76.

Huang, Sui, and Donald E. Ingber. 2007. "A Non-genetic Basis for Cancer Progression and Metastasis: Self-Organizing Attractors in Cell Regulatory Networks." Breast Disease 26 (1): $27-54$.

Huneman, Philippe. 2017. "Outlines of a Theory of Structural Explanations." Philosophical Studies 175 (3): 665-702.

Ingber, Donald E. 2008. "Can Cancer Be Reversed by Engineering the Tumor Microenvironment?" Seminars in Cancer Biology 18:356-64. 
Laforge, Bertrand, David Guez, Michael Martinez, and Jean-Jacques Kupiec. 2005. "Modeling Embryogenesis and Cancer: An Approach Based on an Equilibrium between the Autostabilization of Stochastic Gene Expression and the Interdependence of Cells for Proliferation." Progress in Biophysics and Molecular Biology 89 (1): 93-120.

Levine, Michael, and Eric H. Davidson. 2005. "Gene Regulatory Networks for Development." Proceedings of the National Academy of the Sciences of the USA 102 (14): 4936-42.

National Research Council. 2009. A New Biology for the 21st Century. Washington, DC: National Academies.

Nazarieh, Maryam, Andreas Wiese, Thorsten Will, Mohamed Hamed, and Volkhard Helms. 2016. "Identification of Key Player Genes in Gene Regulatory Networks." BMC Systems Biology 10 (1): 88 .

O’Malley, Maureen A., Ingo Brigandt, Alan C. Love, John W. Crawford, Jack A. Gilbert, Rob Knight, Sandra D. Mitchell, and Forest Rohwer. 2014. "Multilevel Research Strategies and Biological Systems." Philosophy of Science 81 (5): 811-28.

O’Malley, Maureen A., and John Dupré. 2005. "Fundamental Issues in Systems Biology." BioEssays 27 (12): 1270-76.

Sadeghi, Mehdi, Bijan Ranjbar, Mohamad Reza Ganjalikhany, Faiz M. Khan, Ulf Schmitz, Olaf Wolkenhauer, and Shailendra K. Gupta. 2016. "MicroRNA and Transcription Factor Gene Regulatory Network Analysis Reveals Key Regulatory Elements Associated with Prostate Cancer Progression." PLoS One 11 (12): e0168760.

Slamon, D. J., G. M. Clark, S. G. Wong, W. J. Levin, A. Ullrich, and W. L. McGuire. 1987. "Human Breast Cancer: Correlation of Relapse and Survival with Amplification of the HER-2/neu Oncogene." Science 235:177-82.

Udyavar, Akshata R., David J. Wooten, Megan Hoeksema, Mukesh Bansal, Andrea Califano, Lourdes Estrada, Santiago Schnell, Jonathan M. Irish, Pierre P. Massion, and Vito Quaranta. 2017. "Novel Hybrid Phenotype Revealed in Small Cell Lung Cancer by a Transcription Factor Network Model That Can Explain Tumor Heterogeneity." Cancer Research 77 (5): 106374.

Van Laere, Steven, Luc Dirix, and Peter Vermeulen. 2016. "Molecular Profiles to Biology and Pathways: A Systems Biology Approach." Chinese Journal of Cancer 35 (1): 53.

Wagner, Andreas. 2005. "Distributed Robustness versus Redundancy as Causes of Mutational Robustness." Bioessays 27 (2): 176-88.

Weinberg, Robert. 2013. The Biology of Cancer. New York: Garland Science.

- 2014. "Coming Full Circle: From Endless Complexity to Simplicity and Back Again." Cell 157 (1): 267-71.

Yarden, Yosef, and Gur Pines. 2012. "The ERBB Network: At Last, Cancer Therapy Meets Systems Biology." Nature Reviews Cancer 12 (8): 553-63. 